\title{
THE USE OF ARNICA FOR THE Treatment of Soft-Tissue Damage.
}

\begin{abstract}
There is growing anecdotal evidence of the use of homeopathy among sports' participants. Arnica montana [also known as Leopard's Bane] is frequently used as a prophylactic agent both before and after long distance running in the belief that it reduces delayed onset muscle soreness [DOMS]. There is equivocal evidence of its efficacy. The lack of convincing scientific proof is a major reason for homeopathy not being embraced by the medical community. The aim of this review is to discuss the principles of homeopathy in general, followed by a more detailed analysis of the use of arnica in the treatment of soft tissue trauma. Clinical trials published since 1982 were identified using the

\begin{tabular}{|c|}
\hline $\begin{array}{c}\text { BAUER CM, } \\
\text { BSc Physio, BSc MED, B.A.'; } \\
\text { WEIGHT L, PhD'; } \\
\text { LAMBERT MI, PhD'. }\end{array}$ \\
\hline $\begin{array}{l}\text { 'Department of Physiotherapy, } \\
2 \text { University of Chpe Town. } \\
2 \text { Department of Human Biology, University of Cope Town. } \\
{ }^{3} \text { MRC/UCT Research Unit for Exercise Science and Sparts } \\
\text { Medicine, Department of Human Biology, University of } \\
\text { Cope Town, Sports Science Institure of South Africa. }\end{array}$ \\
\hline
\end{tabular}
Medline database. Based on these data it was concluded that there is no overwhelming evidence that treatment with a homeopathic remedy, specifically Arnica montana, consistently reduces the severity of, or the rate of, healing of soft tissue damage.
\end{abstract}

KEY WORDS: HOMEOPATHY, ARNICA TABLETS, DELAYED ONSET MUSCLE SORENESS, LONG DISTANCE RUNNING.

\section{INTRODUCTION}

There is growing anecdotal evidence of the use of homeopathy among sports' participants. For example arnica is frequently used as a prophylactic agent both before and after long distance running in the belief that it reduces delayed onset muscle soreness [DOMS]. Although homeopaths often use arnica for the treatment of soft tissue trauma, there is equivocal evidence of its efficacy (Lokken et al 1995; Jawara et al 1997). This lack of convincing scientific proof is a major reason for homeopathy in general not being accepted by the medical community (Lockie 1998). In contrast the alternative practitioner believes that homeopathy's long history and continued successful use worldwide demonstrates its efficacy.

Despite the differences in approach between homeopathy and conventional

\section{CORRESPONDENCE TO:}

Associate Professor Mike Lambert MRC/UCT Research Unit for Exercise Science and Sports Medicine, P.O. Box 115, Newlands 7925

\section{South Africa}

Tel: (021) 650-4558

Fax: (021) 686-7530

Email: mlambert@sports.uct.ac.za medicine, $40 \%$ of general practitioners in the Netherlands practise homeopathy and $42 \%$ of general practitioners in Britain refer patients to homeopaths (Vallance 1998). In government clinics in India, homeopathy is practiced in conjunction with conventional 'western' medicine (Vallance 1998). According to Jacobs et al (1998) the use of homeopathy is growing in the United States of America.

The aim of this review is to discuss the principles of homeopathy in general, followed by a more detailed analysis of the effectiveness of arnica in the treatment of soft tissue trauma.

\section{ARNICA}

Arnica montana, also known as Leopard's Bane (Allen 1978), is the most frequently studied homeopathic remedy in placebo-controlled trials (Ernst 1998). It is the best known of all homeopathic remedies, and most often used in cases of acute physical trauma to treat both the injury and the accompanying shock (Smith 1998) bruising and post-surgical repair (Hart et al 1997).

Arnica montana is a perennial Alpine herb with a creeping underground stem and a rosette of pale oval leaves. The flowering, erect stem is up to 60 centimeters high, bears a single, bright yellow, daisy-like flower. The plant, which is difficult to cultivate, is native to northern and central Europe and also grows wild in Russia, Scandinavia and northern India (Lawless, 1995).

\section{PRINCIPLES OF HOMEOPATHY}

The word 'homeopathy' is derived from two Greek words, omio meaning 'same' and pathos meaning 'suffering' (Lockie 1998). Homeopathy is regarded as a naturopathic form of medicine (Vallance 1998) that aims to assist the body's healing mechanisms rather than override them (Lockie 1998).

The fundamental premise of the discipline is that a homeopathic remedy, when given to a healthy person, will produce the same symptoms as those of the ill person. The homeopathic remedy stimulates the body's innate healing ability and thereby provokes the body's system to combat these symptoms. This is analogous to the immunizations of conventional medicine that use dilutions of allergens to control the allergies themselves.

\section{NOMENCLATURE}

Homeopathic treatments are prescribed as a " $\mathrm{D}$ "[or " $\mathrm{X}$ "], or " $\mathrm{CH}$ " [centesimal Hahnemann] preparations. "CH" refers to the centesimal scale of the medicinal 
preparation where the original remedy has been diluted on a scale of one drop to 99 drops of water [1 part per 100 parts] and shaken by a process called succussion.

In the case of a $\mathrm{CH} 30$ preparation the whole process is repeated 30 times. The " $D$ " [or " $X$ "] prescription refers to a decimal scale where each dilution involves 9 drops of water to one drop of the original substance (Kaplan 1994). For example a D6 $(6 \mathrm{X})$ is a 1 in 10 dilution repeated 6 times which is obtainable without prescription. D30, [30X] represents a medium potency [dilution $1: 10$ to the power of 30 , that is, succussion repeated 30 times]. According to homeopathic theory the higher the potency the greater the effect. The most commonly used dilution are $30 \mathrm{X}$ preparations (Lokken et al 1995).

\section{THE BASIS OF HOMEOPATHY}

The two main principles of homeopathy are the "simillimum" and "potentisation by succussion" (Reilly et al 1986). According to these principles, if the toxic effects of an agent closely mimic a patient's symptoms, the simillimum argument applies and the physiological reaction provoked by that substance in diluted and succussed amounts may aid the patient's recovery. Analogous to vaccination and immunotherapy, the simillimum principle is sometimes seen as a paradoxical drug effect. The patient is often sensitive to a homeopathic stimulus, which can aggravate symptoms initially. The principle of "potentisation by succussion" applies when the remedy is administered after an initial process of serial dilutions and succussion. The effect of the remedy may be maintained and even enhanced at "apparently absurd dilutions", [ultra-high dilutions (UHDs)] where theoretically none of the original substance remains due to the dilution and succussion process (Reilly et al 1986; Vallance 1998).

Homeopathic remedies are derived primarily from plants, minerals and metals. Substances are tested on healthy human volunteers to determine their therapeutic value. These tests are known as "provings". A prescription is only considered to be effective if the symptoms produced by the remedy during the "provings" match changes in the health of the individual (Smith 1998). This is the basic principle of homeopathy - similia similibus curentur - like cures like. Each homeopathic substance can be appropriately used in a range of conditions, so there are a number of remedies to chose from. Conversely, a single remedy can target a wide variety of conditions. This explains the administration of commonly used, broad-based, over-the-counter remedies for a variety of conditions.

Homeopathy is based on individualized treatment, where ideally a single homeopathic medication is selected according to the signs and symptoms, temperament, disposition, personal and family history of the patient (Lokken et al 1995; Smith 1998).

\section{RESEARCH IN HOMEOPATHY Homeopathy in General}

Research on the efficacy of homeopathic remedies has been an ongoing process for over two hundred years (Koehler 1986). A summary of the clinical trials published since 1982 (identified using the Medline database) is shown in Table 1. The studies have been summarized according to the research design, the dosage and duration of treatment, the outcome variables and the results of the study. Twelve of the 14 studies included a placebo group. Of the remaining two studies, (no placebo group), one study showed that pharmacotherapy was not more effective than homeopathic treatment (Hitzenberger et al 1982) and the other study demonstrated no significant difference in bleeding times immediately following the administration of Arnica montana in a 2-period cross-over trial in healthy volunteers (Baillargeon et al 1993). Ten studies showed that the homeopathic treatment had no advantage over the placebo treatment.

The studies with an objective overview or meta-analysis design are shown in Table 2. These studies are summarized under the headings of 'study selection criteria' and 'general conclusions'. Only one of these studies (Reilly et al 1994] showed that treatment with homeopathy was more effective than treatment with placebo. Two other studies (Kleijnen et al 1991;
Barnes et al 1997) showed that homeopathic remedies tended towards being more effective than placebo, but the remaining six studies concluded that there was insufficient evidence to support any claims of homeopathic efficacy.

Therefore, it can be concluded that there is no overwhelming evidence that treatment with a homeopathic remedy, even Arnica montana reduces the severity of tissue damage or increases the rate of healing.

\section{Effects of arnica on delayed onset muscle sore- ness (DOMS)}

Tveiten et al (1991) assessed the effect of Arnica montana D30 on muscle stiffness, restitution time and muscle cell damage using a double-blind randomized trial following the 1990 Oslo Marathon. Blood tests were carried out before and immediately after the finish of the event, and again after 48 and 72 hours. There were differences in only two of the variables measured between the groups immediately after the finish or after 48 hours and 72 hours. The placebo group had a higher level of plasma creatine kinase [a physiological indication of muscle cell damage] 48 hours post-race. The placebo group also reported experiencing a greater degree of stiffness on all four occasions. The trial indicated that arnica did not reduce the time of restitution but seemed to reduce muscle soreness.

Jawara et al (1997) studied the effects of arnica and rhus tox on DOMS following bench stepping exercise. The authors suggested that homeopathy was an effective treatment although the data were not statistically different. Vickers et al (1997) also compared the effect of a homeopathic preparation of arnica and rhus tox $\mathrm{CH} 30$ and a placebo on DOMS following bench stepping. Their trial also showed that there was no difference between the homeopathic groups and the placebo group in altering the perception of muscle soreness over the five-day period.

In a further study Vickers et al (1998) conducted a randomized, double-blind, placebo-controlled trial to determine whether treatment with homeopathic arnica $30 \mathrm{X}$ was superior to placebo for decreasing muscle soreness following 
Table 1: REVIEW OF INDIVIDUAL TRIALS.

\begin{tabular}{|c|c|c|c|c|c|}
\hline $\begin{array}{c}\text { SUBJECTS } \\
{[\mathrm{M} / \mathrm{F}]}\end{array}$ & $\begin{array}{c}\text { RESEARCH } \\
\text { DESIGN }\end{array}$ & $\begin{array}{l}\text { DOSAGE AND } \\
\text { DURATION }\end{array}$ & $\begin{array}{l}\text { VARIABLES } \\
\text { MEASURED }\end{array}$ & RESULTS & REFERENCE \\
\hline $\begin{array}{l}n=10 \\
{[\mathrm{~m} / \mathrm{f} ?]}\end{array}$ & $\begin{array}{l}\text { Randomized } \\
\text { double blind } \\
\text { cross-over }\end{array}$ & $\begin{array}{l}\text { Patients with essential hypertension } \\
\text { treated with antihypertensive } \\
\text { pharmacotherapy or homeopathic } \\
\text { treatment }\end{array}$ & Blood pressure & $\begin{array}{l}\text { No superiority of } \\
\text { pharmacotherapy over } \\
\text { homeopathic treatment in } \\
\text { decreasing blood pressure }\end{array}$ & $\begin{array}{l}\text { Hitzenberger, } \\
\text { [1982] }\end{array}$ \\
\hline $\begin{array}{l}n=118 \\
{[m / f ?]}\end{array}$ & $\begin{array}{l}\text { Randomized } \\
\text { double blind } \\
\text { placebo } \\
\text { controlled } \\
\text { cross-over }\end{array}$ & $\begin{array}{l}\text { Following surgical removal of } \\
\text { impacted wisdom teeth, under } \\
\text { general anaesthetic } \\
\text { Oral administration } 2 \times \text { day of } \\
\text { Group } 1 \text { Metronidazole } 400 \mathrm{mg} \\
\text { Group 2 Arnica montana } 200 \mathrm{mg} \\
\text { Group 3 Placebo } \quad 1 \text { tablet }\end{array}$ & $\begin{array}{l}\text { Pain control on VAS } \\
\text { Trismus [limitation } \\
\text { of mouth opening] } \\
\text { Prevention of } \\
\text { swelling } \\
\text { Promotion of } \\
\text { healing }\end{array}$ & $\begin{array}{l}\text { Metronidazole greater effect in } \\
\text { pain control, preventing swelling, } \\
\text { and more effective in promoting } \\
\text { healing than arnica and placebo. } \\
\text { Arnica group had greater pain } \\
\text { [p<0.05] and more swelling } \\
\text { than placebo [ } p<0.01]\end{array}$ & $\begin{array}{l}\text { Kaziro, } \\
\text { [1984] }\end{array}$ \\
\hline $\begin{array}{l}n=108 \\
{[m / f ?]}\end{array}$ & $\begin{array}{l}\text { Randomized } \\
\text { double blind } \\
\text { placebo } \\
\text { controlled }\end{array}$ & $\begin{array}{l}\text { Patients with active hayfever given } \\
\text { al week run-in, baseline placebo, } \\
\text { for analysis. Then } 1 \text { tablet placebo } \\
\text { or homeopathic test drug } \\
\text { [ } 30 \mathrm{C} \text { potency] for } 2 \text { weeks, } \\
\text { followed by } 2 \text { weeks observation }\end{array}$ & $\begin{array}{l}\text { Daily VAS of over- } \\
\text { all symptoms and } \\
\text { intensity of sneezing, } \\
\text { blocked and runny } \\
\text { nose, and watery, } \\
\text { red and runny nose. } \\
\text { Similar details } \\
\text { recorded by doctor } \\
\text { at weeks } 0,3 \text { and } 5\end{array}$ & $\begin{array}{l}\text { Subjects treated with homeopathy } \\
\text { had a significant reduction in } \\
\text { symptom scores assessed by } \\
\text { patient and doctor in week five } \\
\text { [final week] } \mathrm{p}=0.02 \text {. } \\
\text { Initial aggravation of symptoms } \\
\text { in homeopathic group followed } \\
\text { by improvement }\end{array}$ & $\begin{array}{l}\text { Reilly et al, } \\
\text { [1986] }\end{array}$ \\
\hline $\mathrm{n}=?$ & $\begin{array}{l}\text { Randomized } \\
\text { double blind } \\
\text { placebo } \\
\text { controlled }\end{array}$ & 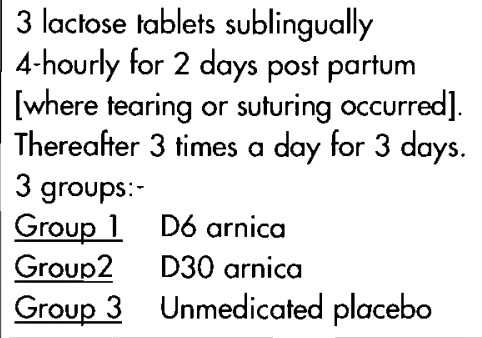 & $\begin{array}{l}\text { Perineal pain } \\
\text { Breast pain } \\
\text { Mood a] Mother } \\
\quad \text { b] Baby } \\
\text { Perineal } \\
\text { appearance }\end{array}$ & $\begin{array}{l}\text { More subjects using arnica D30 } \\
\text { described themselves as 'unhappy' } \\
\text { (p<0.05]. The questionnaire } \\
\text { responses showed a tendency } \\
\text { towards more favourable results } \\
\text { and with arnica D6 than } \\
\text { placebo less favourable with } \\
\text { D30 than placebo }\end{array}$ & $\begin{array}{l}\text { Hofmeyr et al, } \\
\text { [1990] }\end{array}$ \\
\hline $\begin{array}{l}n=36 \\
{[m / f ?]}\end{array}$ & $\begin{array}{l}\text { Randomized } \\
\text { double blind } \\
\text { placebo } \\
\text { controlled }\end{array}$ & $\begin{array}{l}\text { Arnica } C 30 \text {. Five tablets twice } \\
\text { daily for } 5 \text { days starting before } \\
42.2 \mathrm{~km} \text { race. }\end{array}$ & $\begin{array}{l}\text { Blood tests before } \\
\text { race, at finish, } \\
48 \text { hours and } 72 \\
\text { hours after race. } \\
\text { Stiffness evaluated } \\
\text { on [VAS] after finish } \\
\text { and for next } 3 \text { days }\end{array}$ & $\begin{array}{l}\text { No difference in the liver enzymes } \\
\text { or creatine, haptoglobin or } \\
\text { magnesium. Plasma CK increased } \\
\text { in both groups but to a greater } \\
\text { level in placebo group. Difference } \\
\text { greatest on day } 2 \text { [ } p=0.07 \text { ] } \\
\text { A feeling of stiffness more } \\
\text { pronounced in placebo group on } \\
\text { all } 4 \text { tests [ } p=0.06 \text { and } 0.07 \text { on } \\
\text { day } 2 \text { and } 3 \text { ]. No indication that } \\
\text { arnica decreased time of restitution }\end{array}$ & $\begin{array}{l}\text { Treilen et al, } \\
\text { [1991] }\end{array}$ \\
\hline $\begin{array}{l}n=101 \\
{[m=66} \\
f=35]\end{array}$ & $\begin{array}{l}\text { Randomized, } \\
\text { double blind } \\
\text { placebo } \\
\text { controlled } \\
3 \text { groups } \\
\text { [athletics } \\
\text { injuries] }\end{array}$ & $\begin{array}{l}\text { Group } 1 \text { Traumeel S ointment } \\
\text { Group } 2 \text { Traumeel Sine ointment. } \\
\text { [Both contain } 1.5 \mathrm{~g} \text { of arnica D3 } \\
\text { in } 100 \mathrm{~g} \text { ointment] } \\
\text { Group } 3 \text { Placebo. No arnica. } \\
\text { Ointment base without the } \\
\text { Medicinally active ingredients]. } \\
\text { First medication not later than day } \\
4 \text { post injury. Thereafter self- } \\
\text { application twice daily until day } \\
15.6 \text { to } 10 \mathrm{mg} \text { each application }\end{array}$ & $\begin{array}{l}\text { Primary criteria - } \\
\text { abatement } \\
\text { of swelling and } \\
\text { normalisation of } \\
\text { skin temperature } \\
\text { Secondary criteria - } \\
\text { 1] maximum } \\
\text { muscle force } \\
\text { 2] pain index } \\
\text { 3] time interval } \\
\text { for resumption of } \\
\text { training without } \\
\text { complaints }\end{array}$ & $\begin{array}{l}\text { No difference between two } \\
\text { Traumeel ointments when tested } \\
\text { on 5th and 15th day } \\
\text { Difference [p<.0001] between } \\
\text { these and placebo on 15th Day } \\
\text { 1] Maximum muscle force: } \\
\text { Both Traumeel groups superior to } \\
\text { placebo on day } 15 \text { but not day } 5 \\
\text { 2] Pain index: Both Traumeel } \\
\text { groups superior to placebo } \\
\text { day } 5 \text { and } 15 \\
\text { 3] Resume training: Both Traumeel } \\
\text { groups superior to placebo }\end{array}$ & $\begin{array}{l}\text { Bohmer and } \\
\text { Ambrus, } \\
\text { [1992] }\end{array}$ \\
\hline
\end{tabular}


Table 1: REVIEW OF INDIVIDUAL TRIALS.

Continued from page 36.

\begin{tabular}{|c|c|c|c|c|c|}
\hline $\begin{array}{l}\text { SUBJECTS } \\
{[\mathrm{M} / \mathrm{F}]}\end{array}$ & $\begin{array}{l}\text { RESEARCH } \\
\text { DESIGN }\end{array}$ & $\begin{array}{l}\text { DOSAGE AND } \\
\text { DURATION }\end{array}$ & $\begin{array}{l}\text { VARIABLES } \\
\text { MEASURED }\end{array}$ & RESULTS & REFERENCE \\
\hline $\mathrm{n}=?$ & $\begin{array}{l}\text { 2-period } \\
\text { cross-over } \\
\text { trial }\end{array}$ & $\begin{array}{l}\text { Arnica Montana in healthy } \\
\text { volunteers }\end{array}$ & $\begin{array}{l}\text { Bleeding times } \\
\text { and the impact on } \\
\text { various blood } \\
\text { coagulation tests } \\
\text { immediately } \\
\text { following } \\
\text { administration }\end{array}$ & No significant effect & $\begin{array}{l}\text { Baillargeon } \\
\text { et al, } \\
\text { [1993] }\end{array}$ \\
\hline $\begin{array}{l}n=28 \\
{[m / F]}\end{array}$ & $\begin{array}{l}\text { Randomized } \\
\text { double blind } \\
\text { placebo } \\
\text { controlled }\end{array}$ & $\begin{array}{l}4 \text { weeks, single blind placebo. } \\
\text { Then daily dosage for } 8 \text { weeks- } \\
\text { Group I oral homeopathic } \\
\text { immunotherapy to their principal } \\
\text { allergen } \\
\text { Group } 2 \text { identical placebo } \\
\text { [no homeopathic substance] }\end{array}$ & $\begin{array}{l}\text { Daily VAS of overall } \\
\text { symptom intensity }\end{array}$ & $\begin{array}{l}\text { Difference in favour of } \\
\text { homeopathic immunotherapy } \\
\text { within } 1 \text { week of treatment and } \\
\text { persisting up to } 8 \text { weeks } \\
{[p=0.003]}\end{array}$ & $\begin{array}{l}\text { Reilly et al, } \\
\text { [1994] } \\
\text { See meta- } \\
\text { analysis table }\end{array}$ \\
\hline $\begin{array}{l}\mathrm{n}=24 \\
{[\mathrm{~m}=4} \\
\mathrm{f}=20]\end{array}$ & $\begin{array}{l}\text { Randomized } \\
\text { double blind } \\
\text { placebo } \\
\text { controlled } \\
\text { cross-over }\end{array}$ & $\begin{array}{l}3 \text { tablets [containing } 6 \text { homeopathic } \\
\text { drugs at D30 potency, including } \\
\text { arnica] or placebo given } 3 \text { hours } \\
\text { post op. for surgery on } 1 \text { side for } \\
\text { bilateral impacted wisdom teeth } \\
\text { and continued for } 5 \text { days. Following } \\
\text { identical surgical procedure on } \\
\text { opposite side } 14 \text { to } 51 \text { days later, } \\
\text { crossover tablets administered }\end{array}$ & $\begin{array}{l}\text { Pain on VAS } \\
\text { Swelling, } \\
\text { trismus, } \\
\text { and bleeding }\end{array}$ & $\begin{array}{l}\text { No positive evidence for efficacy } \\
\text { of homeopathic treatment on pain } \\
\text { and other inflammatory events }\end{array}$ & $\begin{array}{l}\text { Lokken et al, } \\
\text { [1995] }\end{array}$ \\
\hline $\begin{array}{l}n=60 \\
{[m / f ?]}\end{array}$ & $\begin{array}{l}\text { Randomized } \\
\text { double blind } \\
\text { placebo } \\
\text { controlled }\end{array}$ & $\begin{array}{l}\text { First month baseline, all patients } \\
\text { on placebo. } \\
\text { Thereatter test group on } \\
\text { Individualized homeopathic } \\
\text { Prophylaxis }\end{array}$ & $\begin{array}{l}\text { Frequency and } \\
\text { severity of } \\
\text { migraine attacks }\end{array}$ & $\begin{array}{l}\text { No difference at baseline. } \\
\text { No difference between placebo } \\
\text { and homeopathic group } \\
\text { thereafter }\end{array}$ & $\begin{array}{l}\text { Whitmarsh } \\
\text { et al, } \\
\text { [1997] }\end{array}$ \\
\hline $\begin{array}{l}n=73 \\
{[m=0} \\
f=73]\end{array}$ & $\begin{array}{l}\text { Randomized } \\
\text { double blind } \\
\text { placebo } \\
\text { controlled }\end{array}$ & $\begin{array}{l}2 \text { doses arnica C } 30 \text { tablets or } \\
\text { placebo } 24 \text { hours pre-op. } \\
\text { Then the morning affer total } \\
\text { abdominal hysterectomy, } \\
3 \text { doses/day for } 5 \text { days of arnica } \\
\text { or placebo }\end{array}$ & $\begin{array}{l}\text { Pain and discomfort } \\
\text { on VAS every } \\
12 \text { hours beginning } \\
12 \text { hours pre-op. } \\
\text { Maximum } 10 \\
\text { assessments per } \\
\text { patient. }\end{array}$ & $\begin{array}{l}\text { No difference between placebo } \\
\text { and homeopathic group on } \\
\text { postoperative recovery }\end{array}$ & $\begin{array}{l}\text { Hart et al, } \\
\text { [1997] }\end{array}$ \\
\hline $\begin{array}{l}n=50 \\
{[m / f ?]}\end{array}$ & $\begin{array}{l}\text { Randomized } \\
\text { double blind } \\
\text { placebo } \\
\text { controlled }\end{array}$ & $\begin{array}{l}1 \text { tablet arnica C } 30 \text { and rhus tox } \\
\text { C } 30 \text { ] orally } 3 \text { times a day } 24 \text { hours } \\
\text { prior to bench stepping exercise. } \\
\text { Continued until subject felt no } \\
\text { muscle soreness } \\
\text { Placebo group }\end{array}$ & $\begin{array}{l}\text { DOMS evaluated } \\
\text { on VAS scale every } \\
12 \text { hours for } 7 \text { days }\end{array}$ & $\begin{array}{l}\text { No difference between placebo } \\
\text { and homeopathic group }[p>0.2]\end{array}$ & $\begin{array}{l}\text { Jawara et al } \\
\text { [1997] }\end{array}$ \\
\hline $\begin{array}{l}n=67 \\
{[m=23} \\
f=34]\end{array}$ & $\begin{array}{l}\text { Randomized, } \\
\text { double blind } \\
\text { placebo } \\
\text { controlled }\end{array}$ & $\begin{array}{l}1 \text { tablet } 3 x / \text { day orally of a complex } \\
\text { of arnica } C 30 \text {, rhus tox } C 30 \text { and } \\
\text { Sarcolactic acid } \\
\text { Placebo group }\end{array}$ & $\begin{array}{l}\text { Muscle soreness } \\
\text { scored on Likert } \\
\text { scale } 5 \text { days after } \\
10 \text { minute bench- } \\
\text { stepping exercise }\end{array}$ & $\begin{array}{l}\text { No difference between placebo } \\
\text { and homeopathic group }\end{array}$ & $\begin{array}{l}\text { Vickers } \\
\text { et al, } \\
\text { [1997] }\end{array}$ \\
\hline $\begin{array}{l}n=400 \\
{[\mathrm{~m} / \mathrm{f} ?]}\end{array}$ & $\begin{array}{l}\text { Randomized, } \\
\text { double blind } \\
\text { placebo } \\
\text { controlled }\end{array}$ & $\begin{array}{l}\text { Arnica C } 30 \text { group } \\
\text { Placebo group }\end{array}$ & $\begin{array}{l}\text { Muscle soreness } \\
\text { scored twice daily } \\
\text { on Likert scale for } \\
\text { the } 5 \text { days following } \\
\text { long distance racing }\end{array}$ & $\begin{array}{l}\text { No difference between placebo } \\
\text { and homeopathic group }\end{array}$ & $\begin{array}{l}\text { Vickers } \\
\text { et al, } \\
\text { [1998] }\end{array}$ \\
\hline
\end{tabular}

Abbreviations:

$m=$ male, $f=$ female, VAS $=$ visual analogue scale, $\quad ?=$ data unavailable, DOMS = delayed onset muscle soreness 
Table 2: REVIEW OF META-ANALYSES (From 1990).

\begin{tabular}{|c|c|c|c|}
\hline NO. OF STUDIES & STUDY SELECTION CRITERIA & CONCLUSIONS & REFERENCE \\
\hline $\begin{array}{l}\mathrm{n}=40 \\
\text { (total of } 523 \text { subjects) }\end{array}$ & $\begin{array}{l}40 \text { published randomised trials: } \\
\text { results of the homeopathic } \\
\text { treatment were compared to } \\
\text { those of a standard treatment, } \\
\text { placebo, or no treatment }\end{array}$ & $\begin{array}{l}\text { Results did not provide acceptable evidence that } \\
\text { homeopathic treatments are effective }\end{array}$ & $\begin{array}{l}\text { Hill and Doyon, } \\
\text { [1990] }\end{array}$ \\
\hline $\mathrm{n}=107$ & & $\begin{array}{l}81 \text { of } 105 \text { trials with interpretable results, } \\
\text { were positive regardless of the quality of the trial } \\
\text { or the variety of homeopathy used. Evidence of } \\
\text { clinical trials is positive, but not sufficient to draw } \\
\text { definite conclusions because most trials were } \\
\text { of low methodological quality and unknown } \\
\text { publication bias }\end{array}$ & $\begin{array}{l}\text { Kleijnen et al, } \\
{[1991]}\end{array}$ \\
\hline$n=213$ & & $\begin{array}{l}\text { Medline and Embase searches give an } \\
\text { "impression" of the evidence }\end{array}$ & $\begin{array}{l}\text { Kleiinen and } \\
\text { Knipschild, } \\
\text { [1992] }\end{array}$ \\
\hline $\begin{array}{l}n=3 \\
\text { (total of } 202 \text { subjects) }\end{array}$ & & $\begin{array}{l}\text { The effects of homeopathy were greater than } \\
\text { placebo }[p=0.0004]\end{array}$ & $\begin{array}{l}\text { Reilly et al, } \\
\text { [1994] }\end{array}$ \\
\hline $\begin{array}{l}n=6 \\
n=776 \text { [cases] }\end{array}$ & & $\begin{array}{l}\text { Evidence that homeopathic remedies }=/<12 \mathrm{C} \\
\text { [but not }=/>12 \mathrm{C}] \text { can reduce time to first } \\
\text { flatus after abdominal or gynaecological } \\
\text { surgery }[p<0.05]\end{array}$ & $\begin{array}{l}\text { Barnes et al, } \\
\text { [1997] }\end{array}$ \\
\hline $\mathrm{n}=89$ & & Insufficient evidence that homeopathy is effective & Linde et al, [1997] \\
\hline $\begin{array}{l}n=32 \\
\text { (total of } 1778 \text { subjects)] }\end{array}$ & & $\begin{array}{l}\text { Individualized homeopathy has effect over placebo. } \\
\text { Evidence however, not convincing - methodological } \\
\text { shortcomings and inconsistencies }\end{array}$ & $\begin{array}{l}\text { Linde and } \\
\text { Melchart, } \\
\text { [1998] }\end{array}$ \\
\hline$n=8$. & & $\begin{array}{l}\text { The claim that homeopathic arnica is effective over } \\
\text { placebo is not supported by rigorous clinical trials }\end{array}$ & $\begin{array}{l}\text { Ernst and } \\
\text { Pittler, [1998] }\end{array}$ \\
\hline $\begin{array}{l}\mathrm{n}=? \\
\text { (not described) }\end{array}$ & & $\begin{array}{l}\text { Homeopathy perceived to be ineffective for any } \\
\text { type of low blood pressure }\end{array}$ & $\begin{array}{l}\text { Ernst and } \\
\text { Pittler, [1999] }\end{array}$ \\
\hline
\end{tabular}

long distance running. Four hundred subjects completed a visual analog and Lickert scale of muscle soreness twice daily for the five days following their race. The authors concluded from their results that arnica was not effective in reducing muscle soreness after long distance running (Vickers et al, 1998).

\section{DISCUSSION AND CONCLUSION}

The aim of this review was to describe the general principles of homeopathy followed by an analysis of the research on the efficacy of homeopathic treatment, specifically Arnica montana. It is clear from the data in Tables 1 and 2 that there is no convincing evidence that treatment with a homeopathic remedy consistently reduces the severity of, or increases the rate of healing of damaged tissue.

Some homeopathic remedies are diluted to the point where there can be no remaining molecules present to explain their proposed biological effects. The use of Ultra High Dilutions [UHDs] appears to many scientists to make homeopathy a scientific absurdity. According to Vallance (1998) most scientists reject UHD effects because of their intrinsic implausibility in the light of current scientific understanding. Lokken et al (1995) question whether the infinitesimally diluted substances used in homeopathy really exert biological activity and Vandenbroucke (1997) argues that the 'infinite dilutions' of the agents used cannot possibly produce any measurable effect. Their scepticism is supported by the absence of any scientific proof of such activity (Lockie 1998). Yet others, such as Endler and Schulte (1994), believe that UHDs have an effect, relying on the accepted homeopathic concept of 'hormesis', the belief that high concentrations of a homeopathic agent suppress, while low ones stimulate healing. In an editorial comment, Davenas et al (1988) uses the 
argument that an aqueous solution of a homeopathic substance retains its ability to elicit a biological response even at such high dilutions where there is negligible chance of a single molecule remaining in any sample. This is based on the concept that dilutions are followed by vigorous shaking [succussion], and the transmission of the biological information could be related to the molecular organization of water (Davenas et al 1988).

The studies evaluated in table 1 and 2 were designed according to the classic scientific rationale of an experimental group receiving the treatment, and a control group receiving a placebo. However, this goes against the basic edict of homeopathy, where prescriptions are highly individualized to meet the needs of the patient. As Koehler (1986) points out, double-blind trials are unacceptable for establishing the efficacy of homeopathic remedies because, in accordance with homeopathic principles, the individual reactivity and receptiveness of the subject must be taken into account and the dose attenuated accordingly. According to Rivett (1999) double-blind, placebo-controlled clinical trials should not be regarded as the only acceptable evidence of a treatment or drug's therapeutic value. Smith (1998) is of the opinion that the inappropriateness of the randomized clinical trial model for the individualized prescription is now being overcome with the development of new double-blind protocols that are more patient orientated.

In summary, scientists are taught to evaluate evidence according to a set of rules (double blind placebo type studies). Homeopathy, due to the reasons described, precludes an evaluation using a double blind placebo design. To be examined and judged by the scientific process, an alternative system to the conventional system must be used. Until this happens, homeopathy will be viewed with scepticism by scientists. At present scientists have proved (using their rules) that homeopathy does not work. The responsibility would appear to be that of the homeopaths to establish a set of rules that is acceptable to the scientific community and which can be used to evaluate homeopathic treatment.

\section{REFERENCES}

Allen HC 1978 Keynotes and Characteristics with Comparisons of some of the leading Remedies of the Materia Medica. Thorsons, Northamptonshire

Baillargeon L, Drouin J, Desjardins L, Leroux D, Audel D 1993 The effects of Arnica Montana on blood coagulation. Randomized controlled trial. Canadian Family Physician 39:2362-2367

Barnes J, Resch KL, Ernst E 1997 Homeopathy for postoperative ileus? A metaanalysis. Journal of Clinical Gastroenterology 25:628-633

Bohmer D, Ambrus P 1992 Treatment of Sports Injuries with Traumeel Ointment: A Controlled Double-Blind Study with Traumeel Ointment for Treatment of Sports Injuries. Biological Therapy X:4

Davenas E, Beauvais F, Amara J, Oberbaum M, Robinzon B, Miadonna A, Tedeschi A, Pomeranz B, Fortner P, Belon P, Sainte-Laudy J, Poitevin B, Benveniste J 1988 Human basophil degranulation triggered by very dilute antiserum against IgE. Nature 333: 816-818

Endler PC, Schulte J (eds)1994 Ultrahigh dilution: Physiology and physics. Kluwer Academic, Dordrecht

Ernst E, Pittler MH 1998 Efficacy of homeopathic arnica. A systematic review of placebo controlled clinical trials. Archives of Surgery 133:1187-1190

Ernst E, Pittler MH 1999 Experts' opinions on complementary /alternative therapies for low back pain. Journal of Manipulative Physiological Therapeutics 2:87-90

Hart O, Mullee MA, Lewith G, Miller J 1997 Double-blind, placebo-controlled, randomized clinical trial of homeopathic arnica C30 for pain and infection after total abdominal hysterectomy. Journal of the Royal Society of Medicine 90:73-78

Hill C, Doyon F 1990 Review of randomized trials of homeopathy. Reveu Epidemiology Sante Publique 38:139-147

Hitzenberger G, Korn A, Dorcsi M 1982 A controlled randomized double-blind cross-over study of the effects of antihypertensive pharmacotherapy and homeopathy in patients with essential hypertension. Wien-Klin-Wochenschr 91:665-670

Hofmeyr GJ, Piccioni V, Blauhof P 1990 Postpartum homeopathic Arnica montana: a potency-finding study. British Journal of Clinical Practise 44:619-621

Jacobs J, Chapman EH, Crothers D 1998 Patient characteristics and practice patterns of physicians using homeopathy. Archives of Family Medicine 7:537-540

Jawara N, Lewith GT, Vickers AJ, Mullee MA, Smith C 1997 Homeopathic arnica and Rhus toxicodendron for delayed onset muscle soreness. British Homeopathic Journal 86: 10-15

Kaplan B 1994 Homeopathy: 2. In pregnancy and for the under-fives. Professional Care Mother Child 4:185-187

Kaziro GSN 1984 Metronidazole (flagyl) and arnica montana in the prevention of postsurgical complications, a comparative placebo controlled clinical trial. British Journal of Oral and Maxillofacial Surgery 22:42-49

Kleijnen J, Knipschild P, ter Riet G 1991 Clinical trials of homeopathy. British Medical Journal 302:316-323

Kleijnen J, Knipschild P 1992 The comprehensiveness of Medline and Embase computer searches. Searches for controlled trials of homeopathy, ascorbic acid for common cold and ginkgo biloba for cerebral insufficiency and intermittent claudication. Pharmacologie Weekblad Science 14:316-320

Koehler G 1986 The Handbook of Homeopathy. Its Principles and Practice. 4thedn. pp I 1-25. Thorsons, Northamptonshire

Lawless J 1995 The Encyclopaedia of Essential Oils. Element Books, Brisbane

Linde K, Clausius N, Ramirez G, Melchart D, Eitel F, Hedges LV, Jonas WB 1997 Are the clinical effects of homeopathy placebo effects? A meta-analysis of placebo-controlled trials. Lancet 350:834-843

Linde K, Melchart D 1998 Randomized controlled trials of individualized homeopathy: a state-of-the-art review. Journal of Alternative Complementary Medicine 4:371-388 
Lockie A 1998 The family guide to Homeopathy. 2nd ed. pp9-16. Hạmish Hamilton, London

Lokken P, Straumsheim PA, Tveiten D, Skjelbred P, Borchgrevink CF 1995 Effect of homeopathy on pain and other events after acute trauma: placebo controlled trial with bilateral oral surgery. British Medical Journal 310:1439-1442

Reilly DT, Taylor MA, McSharry C, Aitchison T 1986 Is homeopathy a placebo response? Controlled trial of homeopathic potency, with pollen in hayfever as model. Lancet 2:881-886

Reilly D, Taylor MA, Beattie NGM, Campbell JH, McSharry C, Aitchison TC, Carter R, Stevenson RD 1994 Is evidence for homeopathy reproducible? Lancet 344:1601-1606
Rivett DA 1999 Manual therapy cults. Manual Therapy 4:125-126

Smith JL 1998 Using homeopathy during pregnancy and labour. Journal of the Association of Chartered Physiotherapists in Women's Health 82:8-10

Tveiten D, Bruseth S, Borchgrevink CF, Lohne K 1991 Effect of Arnica D30 during hard physical exertion. A double-blind randomized trial during the Oslo Marathon. Tidsskr Nor Laegeforen 111: 3630-3631

Vallance AK 1998 Can biological activity be maintained at ultra-high dilution? An overview of homeopathy, evidence, and Bayesian philosophy. Journal of Alternative Complementary Medicine 4:49-76
Vandenbroucke JP 1997 Homeopathy trials: going nowhere. Lancet 350:824

Vickers AJ, Fisher P, Smith C, Wyllie SE, Lewith GT 1997 Homeopathy for delayed onset muscle soreness: a randomized double blind placebo controlled trial. British Journal of Sports Medicine 31:304-307

Vickers AJ, Fisher P, Smith C, Wyllie SE, Rees R 1998 Homeopathic Arnica 30x is ineffective for muscle soreness after longdistance running: a randomized, double-blind, placebo-controlled trial. Clinical Journal of Pain 14:227-231

Whitmarsh TE, Coleston-Shields DM, Steiner TJ 1997 Double- blind randomized placebocontrolled study of homeopathic prophylaxis of migraine. Cephalalgia 17:600-604

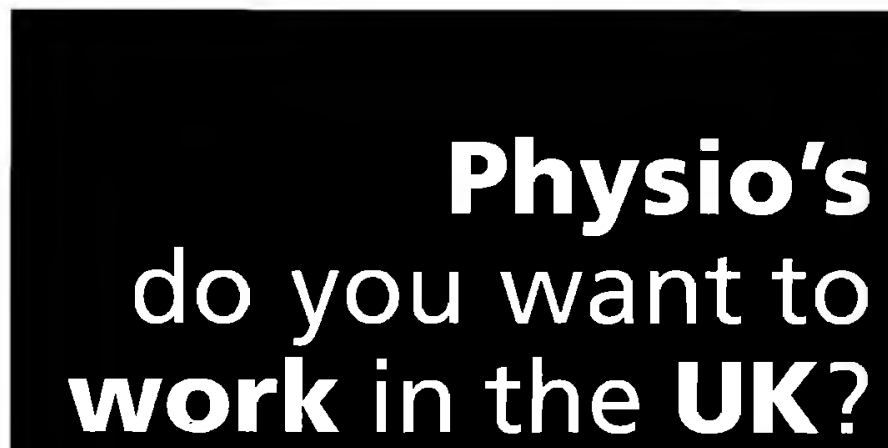

Search our jobs@

www.paramedics-workline.co.uk "Commited to equal opportunities"

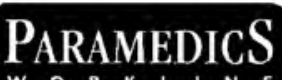

Call Riekie Friedrich now on: (012) 3624523 Fax us your CV on: (012) 3624229 or e-mall: r.friedrichoparamedics-workline.co.uk

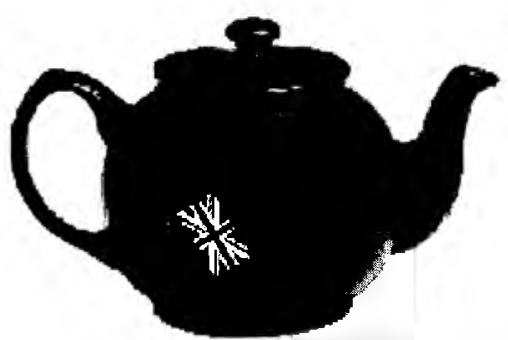

Is working in the UK your cup of tea?

Angela Shaw Associates have over 10 years experience in the successful placement of overseas therapists throughout the UK and Ireland.

If you're a Physiotherapist, Occupational Therapist or Speech and Language Therapist we offer a number of benefits including:

- Free tax advice - CPSM refund ${ }^{*}$ Accident Insurance - Enhanced rates of pay - Temporary and permanent positions Enormous range of opportunities throughout the UK and Ireland $\square$ Training allowance ${ }^{*}$ Holiday pay * Subject to qualifying status

If you're looking to make the move contact one of our dedicated consultants today.

Call free from South Africa today on 0800990767

tel: +44 (0) 2085547691

email: asa@match.co.uk

web: www asa-locums.co.uk a angela shaw associates a member of the match group 